\title{
Atividade física e saúde mental durante a pandemia da COVID-19: uma revisão rápida de estudos epidemiológicos brasileiros
}

\section{Physical activity and mental health during the covid-19 pandemic: a rapid review of Brazilian epidemiological studies \\ Actividad física y salud mental durante la pandemia del covid-19: una revisión rápida de los estudios epidemiológicos brasileños}

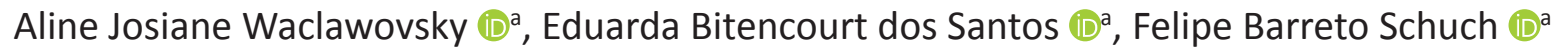
a Universidade Federal de Santa Maria - UFSM, Departamento de métodos e técnicas desportivas - Santa Maria/RS Brasil.
\end{abstract}

Instituição: Universidade Federal de Santa Maria - UFSM

DOI 10.5935/2318-0404.20210011

\section{Resumo}

O distanciamento social, uma das principais estratégias para minimizar a transmissão e o contágio pelo novo coronavírus, provoca um rompimento brusco e substancial nos comportamentos da vida diária, levando a diminuição dos níveis de atividade física e piora dos sintomas de depressão e ansiedade. O objetivo do estudo foi realizar uma revisão rápida de estudos epidemiológicos brasileiros que avaliaram a associação entre atividade física e saúde mental durante a pandemia de COVID-19. A busca pelos estudos foi realizada nas bases Scielo, Scielo Preprints e PubMed até o dia 27 de janeiro de 2021. Foram incluídos seis estudos que avaliaram um total de 64.473 brasileiros, com idades acima dos 18, de todas as regiões do país. Dos estudos analisados, quatro demonstraram que há uma associação entre atividade física e sintomas de depressão e ansiedade, um demonstrou que há associação entre atividade física e depressão e um que há associação entre atividade física e sintomas de ansiedade. Ser fisicamente inativo durante a pandemia da COVID-19 está associado a um risco $152 \%$ maior de apresentar depressão e 118\% maior de ansiedade. Realizar 30 minutos ou mais de atividade física moderada a vigorosa por dia está associado a redução de $29 \%$ no risco de sintomas depressivos, $28 \%$ de ansiedade e $29 \%$ na co-ocorrência de sintomas de depressão e ansiedade. A prática de atividade física é uma 
estratégia importante de saúde pública para mitigar o impacto da pandemia e das medidas de isolamento social na saúde mental da população Brasileira.

Palavras-chaves: Atividade Física; Saúde Mental; COVID-19

\begin{abstract}
Social distancing, the main strategy to minimize transmission and contagion by the coronavirus, causes a sudden and substantial disruption in daily life behavior, leading to decreased levels of physical activity and worsening symptoms of depression and anxiety. The aim of the study was to conduct a rapid review of Brazilian epidemiological studies that evaluated the association between physical activity and mental health during the COVID-19 pandemic. The search for the studies was carried out on the Scielo, Scielo Preprints and PubMed databases until January 27,2021 . Six studies were included that evaluated a total of 64,473 Brazilians, over 18 , from all regions of the country. Of the studies analyzed, four demonstrated an association between physical activity and symptoms of depression and anxiety, one demonstrated that there is an association between physical activity and depressive symptoms and one that there is an association between physical activity and symptoms of anxiety. Being physically inactive during the COVID-19 pandemic is associated with a $152 \%$ higher risk of depression and $118 \%$ greater anxiety. Performing 30 minutes of moderate to vigorous physical activity per day is associated with a $29 \%$ reduction in the risk of depressive symptoms, $28 \%$ anxiety and $29 \%$ in the co-occurrence of symptoms of depression and anxiety. The practice of physical activity is an important public health strategy to mitigate the impact of the pandemic and social isolation measures on the mental health of the Brazilian population.
\end{abstract}

Keywords: Physical activity; Mental health; COVID-19

\title{
Resumen
}

El distanciamiento social, una de las principales estrategias para minimizar la transmisión y contagio por el nuevo coronavirus, provoca una alteración repentina y sustancial en el comportamiento y la vida diaria, lo que lleva a una disminución de los niveles de actividad física y al empeoramiento de los síntomas de depresión y ansiedad. El objetivo del estudio fue realizar una revisión rápida de los estudios epidemiológicos brasileños que evaluaron la asociación entre la actividad física y la salud mental durante la pandemia de COVID-19. La búsqueda de los estudios se realizó en las bases de datos Scielo, Scielo Preprints y PubMed hasta el 27 de enero de 2021. Se incluyeron seis estudios que evaluaron a un total de 64.473 brasileños, mayores de 18 años, de todas las regiones del país. De los estudios analizados, cuatro demostraron que existe asociación entre actividad física y síntomas de depresión y ansiedad, uno demostró que existe asociación entre actividad física y depresión y uno que existe asociación entre actividad física y ansiedad. Estar físicamente inactivo durante la pandemia de COVID-19 se asocia con un 152\% más de riesgo de depresión y un 118\% más de ansiedad. Realizar 30 minutos o más de actividad física de moderada a vigorosa por día se asocia con una reducción del 
$29 \%$ en el riesgo de síntomas depresivos, $28 \%$ de ansiedad y $29 \%$ en la co-ocurrencia de síntomas de depresión y ansiedad. La práctica de actividad física es una importante estrategia de salud pública para mitigar el impacto de la pandemia y las medidas de aislamiento social en la salud mental de la población brasileña.

Palabras clave: Actividad física; Salud mental; COVID-19

\section{Introdução}

No final de 2019, em Wuhan, na China, foi relatado pela primeira vez um surto provocado por um novo vírus, o coronavírus da síndrome respiratória aguda 2 (SARS-COV-2), causador da doença por coronavírus-2019 (COVID-19). No dia 11 de março de 2020, a Organização Mundial da Saúde (OMS), declarou oficialmente o surto do novo Coronavírus (COVID-19) como uma pandemia, provocando uma situação de emergência na saúde global e levando diversos países a se valerem de medidas preventivas para frear o contágio e a transmissão da doença ${ }^{1}$.

Dentre as medidas preventivas, uma das principais recomendações feita pela OMS foi o distanciamento físico, que consiste na limitação da circulação de indivíduos na sociedade, reduzindo seu contato com outras pessoas $^{1}$. Essa medida visa conter a propagação da doença e o consequente impacto grave da pandemia ${ }^{1}$.

No Brasil, houve a orientação sobre a adotar medidas de etiqueta respiratória, higiene de mãos e principalmente de distanciamento social, para reduzir a velocidade de transmissão do vírus ${ }^{2}$. O distanciamento social é uma forma eficaz para diminuir a transmissão do coronavírus, porém ela provoca grandes mudança no estilo de vida e no comportamento humano, alterando o nível de atividade física e o tempo gasto em comportamento sedentário, em níveis populacionais ${ }^{3,4}$. O impacto da pandemia e do isolamento social nos níveis de atividade física pode, no entanto, diferir entre os países. No Reino Unido, alguns estudos sugerem um aumento no tempo gasto em atividade física ${ }^{4}$, enquanto no Brasil os estudos são mais consistentes em mostrar uma redução $5,6,7$.

Durante a pandemia, devido tanto as medidas restritivas, quanto ao medo pandêmico ${ }^{8}$ é esperado um incremento nos sintomas depressivos e de ansiedade em nível populacional ${ }^{9,10}$. Um estudo transversal, realizado com 45.161 participantes brasileiros, identificou que 40,4\% (IC 95\% 39,0 - 41,8) deles sentiram-se tristes ou deprimidos durante o distanciamento social e que 52,6\% (IC 95\% 51,2 - 54,1) relataram sentirem-se ansiosos sempre ou quase sempre durante o isolamento ${ }^{11}$. Em um estudo longitudinal, que acompanhou 2.314 brasileiros, 51,3\% dos participantes relataram piora nos sintomas de ansiedade e um a cada três participantes apresentaram sintomas depressivos agravados durante o período de distanciamento social ${ }^{12}$.

A atividade física, em períodos pré-pandêmicos, é um fator protetor contra a incidência de depressão e ansiedade ${ }^{13,14}$. Uma meta-análise de estudos longitudinais, incluindo dados de mais de 260 mil sujeitos, identificou que atividade física está associada a redução no risco de desenvolver depressão e que pessoas que têm níveis mais altos de atividade física apresentam $17 \%$ menos chances de apresentar depressão quando comparadas com pessoas fisicamente menos ativas ${ }^{13}$. Evidências meta-analíticas de estudos longitudinais, 
incluindo informações de mais de 70 mil sujeitos, demonstraram que pessoas com maiores níveis de atividade física têm $26 \%$ menos chances de desenvolverem ansiedade ${ }^{14}$.

No contexto pandêmico, no qual parece haver piora da saúde mental e uma redução do nível de atividade física da população brasileira, ainda não está claro o quanto o efeito protetor da atividade física sobre a saúde mental se mantém ou é modificado. $O$ objetivo do presente estudo foi realizar uma revisão rápida de estudos epidemiológicos brasileiros que avaliaram a associação entre atividade física e saúde mental durante a pandemia de COVID-19.

\section{Metodologia}

Foi realizada uma busca nas bases Scielo, Scielo Preprints e PubMed até o dia 27 de janeiro de 2021, por estudos realizados no Brasil, que avaliaram a associação entre atividade física, sintomas de depressão e ansiedade durante a pandemia de COVID-19. Para isso, utilizamos a seguinte estratégia de busca: ((physical activity OR exercise) AND (mental health OR depression OR anxiety) AND (covid-19 OR coronavirus) AND (Brazil OR brazilians)), sendo adaptada de acordo com a base. Buscas manuais também foram realizadas no ResearchGate e no Google Scholar, assim como nas referências dos estudos incluídos.

Foram incluídos estudos que: 1) avaliaram e/ou relataram níveis de atividade física dos participantes; 2) relataram sintomas de depressão e ansiedade através de instrumentos que tiveram as suas características psicométricas testadas e validadas; 3 ) testaram a associação entre atividade física e depressão ou ansiedade utilizando modelos de regressão ajustados ou não ajustados por covariáveis ou que compararam a diferença entre as médias de sintomas entre os mais ativos e os menos ativos; 4) foram realizados com a população brasileira (maiores de 18 anos) durante a pandemia de COVID-19; 5) foram escritos em português ou inglês. Excluímos estudos de revisão, editoriais e artigos de opinião.

Os dados extraídos dos estudos foram: primeiro autor, ano de publicação, região do país, tamanho da amostra, características da amostra como média de idade, sexo, nível de atividade física dos participantes e informações sobre sintomas de depressão e ansiedade. Ademais, foram analisados os instrumentos utilizados para avaliação da atividade física e dos sintomas de depressão e ansiedade, e também a relação dos sintomas de depressão e ansiedade com a atividade física.

Após a coleta de dados, foi realizada uma síntese qualitativa dos estudos em virtude da heterogeneidade na apresentação dos dados e impossibilidade de se realizar uma meta-análise.

\section{Resultados}

A busca nas bases de dados retornou 79 artigos e após a remoção dos duplicados, 71 títulos e/ou resumos foram lidos, restando para leitura completa 9 estudos potencialmente elegíveis. As buscas manuais identificaram mais 14 estudos potencialmente elegíveis. Dos 23 estudos potencialmente elegíveis, 6 foram incluídos nesta revisão. $\mathrm{O}$ fluxograma da seleção dos estudos pode ser visto na figura 1. 
Os seis estudos incluídos avaliaram um total de 64.473 brasileiros, com idades acima dos 18 anos, de todas as regiões do país. Dos seis estudos incluídos, um era do tipo longitudinal ${ }^{12}$ e 5 eram do tipo transversal ${ }^{15-19}$. Os níveis de atividade física foram estabelecidos por instrumentos ou questionários de autorrelato, os sintomas de depressão e/ou ansiedade por meio de instrumentos de screening, quatro estudos avaliaram a associação entre atividade física e sintomas de depressão e ansiedade ${ }^{12,17-19}$, um estudo avaliou a associação entre atividade física e sintomas depressivos ${ }^{15}$ e um a associação entre atividade física e ansiedade ${ }^{16}$ conforme apresentado na tabela 1.

\section{Figura 1. Seleção de estudos}

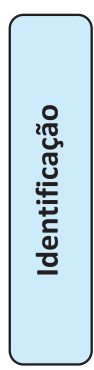

Estudos identificados nas bases de dados $(n=79)$ PubMed $(n=41)$ Scielo / Scielo Preprints $(n=38)$
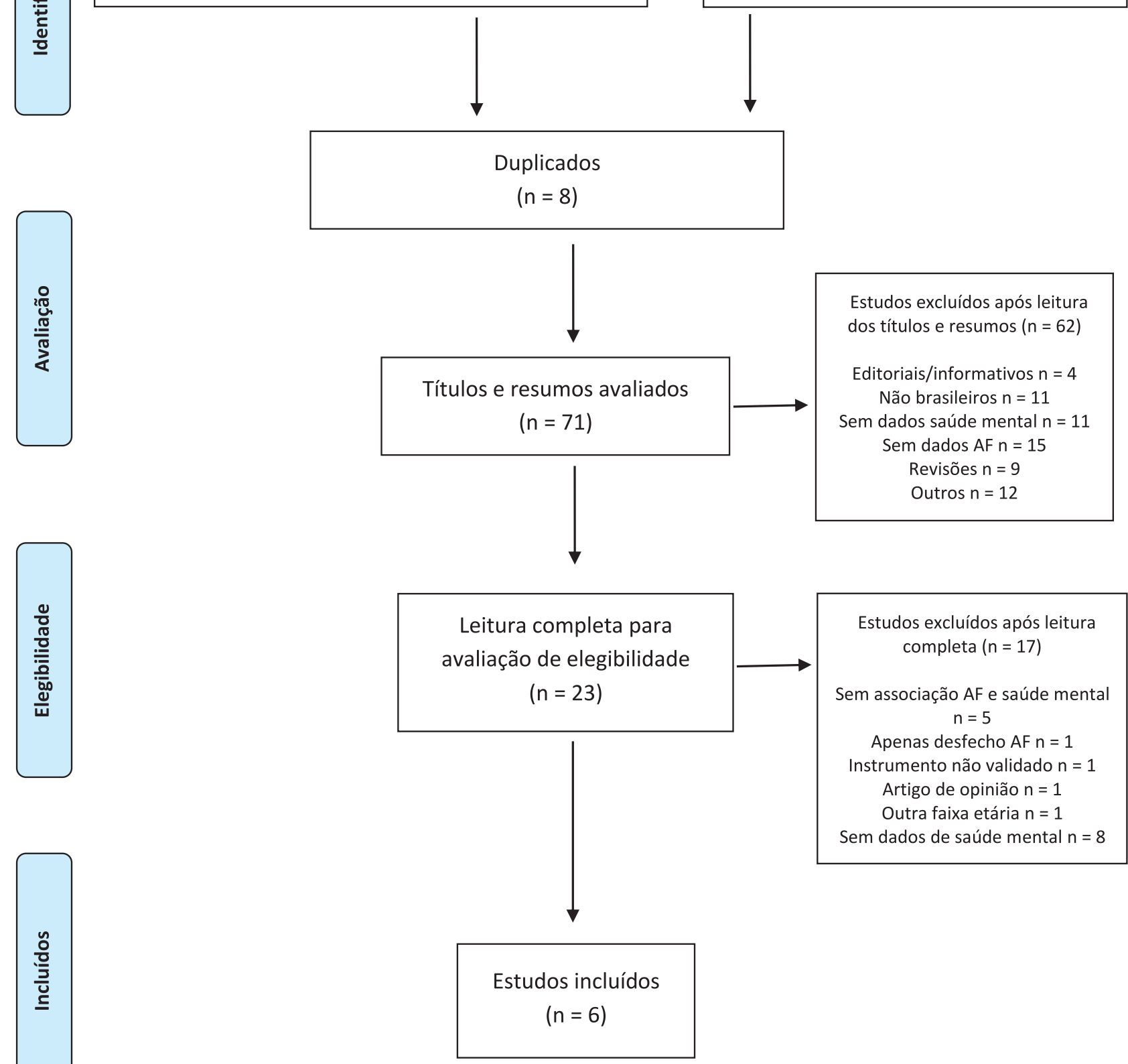
Tabela 1. Caracterização dos estudos incluídos

\begin{tabular}{|c|c|c|c|c|c|c|c|c|c|}
\hline \multirow{2}{*}{$\begin{array}{l}\text { AUTOR/ } \\
\text { ANO }\end{array}$} & \multirow{2}{*}{$\begin{array}{l}\text { REGIÃO } \\
\text { DO PAÍS }\end{array}$} & \multicolumn{3}{|c|}{ AMOSTRA } & \multirow{2}{*}{$\begin{array}{l}\text { Sexo (\% S } \\
\text { feminino) }\end{array}$} & \multirow{2}{*}{$\begin{array}{l}\text { INSTRUMENTO } \\
\text { ATIVIDADE } \\
\text { FÍSICA }\end{array}$} & \multirow{2}{*}{$\begin{array}{l}\text { PREVALÊNCIA DE } \\
\text { SINTOMAS DE } \\
\text { DEPRESSÃO E } \\
\text { ANSIEDADE (D\&A) }\end{array}$} & \multirow{2}{*}{$\begin{array}{l}\text { INSTRUMENTO } \\
\text { SINTOMAS } \\
\text { D\&A }\end{array}$} & \multirow[b]{2}{*}{ RESULTADOS } \\
\hline & & N & Idade & $\begin{array}{l}\text { Sexo (\% S } \\
\text { feminino) }\end{array}$ & & & & & \\
\hline $\begin{array}{l}\text { Andrade- } \\
\text { Lima et. } \\
\text { al, } 2020\end{array}$ & $\begin{array}{l}\text { Todo } \\
\text { Brasil }\end{array}$ & $\begin{array}{l}59.401 \\
\text { adultos }\end{array}$ & $\begin{array}{l}\text { Idade média } \\
\text { de } 43,1 \text { (IC } \\
95 \%: 42.8 \text { a } \\
43.3 \text { ) anos }\end{array}$ & $56,4 \%$ & $\begin{array}{l}\text { Inativo }{ }^{(1)}: 81,2 \% \\
\text { Ativo(2): }^{2}: 18,8 \%\end{array}$ & $\begin{array}{c}3 \text { questões de } \\
\text { autorrelato }\end{array}$ & $\begin{array}{l}\text { Risco de depressão: } \\
\qquad, 3 \%\end{array}$ & PHQ-9 & $\begin{array}{c}\text { Homens inativos sem condição crônica: } \\
\text { OR=1,64 (IC } 95 \%=1,15-2,34 \text { ) de } \\
\text { sintomas depressivos aumentados. } \\
\text { Mulheres inativas sem condição } \\
\text { crônica: } \\
\text { OR=1,16 (IC } 95 \%=0,87-1,54 \text { ) de } \\
\text { sintomas depressivos aumentados }\end{array}$ \\
\hline $\begin{array}{l}\text { Feter et. } \\
\text { al, } 2020\end{array}$ & Região Sul & $\begin{array}{c}2.321 \\
\text { adultos }\end{array}$ & $\begin{array}{c}\text { 31-59 anos: } \\
54,3 \%\end{array}$ & $76,6 \%$ & $\begin{array}{c}\text { Antes do } \\
\text { distanciamento } \\
\text { Inativo(1): } 56,8 \% \\
\text { Ativo }{ }^{2}: 43,2 \% \\
\text { Com } \\
\text { distanciamento } \\
\text { Inativo sustentado - } 46,9 \% \\
\text { Tornou-se inativo - } 26,6 \% \\
\text { Tornou-se ativo - } 8,3 \%\end{array}$ & $\begin{array}{l}\text { Autorrelato } \\
\text { com } 4 \text { questões. }\end{array}$ & $\begin{array}{c}\text { Ansiedade: } \\
\text { Sem mudança - } \\
\quad 44,9 \% \\
\text { Piorando - 51,3\% } \\
\text { Melhor - 3,8\% } \\
\text { Depressão: } \\
\text { Sem mudança - } \\
\quad 60,3 \% \\
\text { Piorando - 35\% } \\
\text { Melhor - 4,7\% } \\
\end{array}$ & HADS & $\begin{array}{c}\text { Mantiveram-se ativos: } \mathrm{RP}=0,72 \text { (IC } 95 \% \\
0,57-0,91 \text { ) de sintomas depressivos; } \\
\text { Tornaram-se ativos: } \mathrm{RP}=0,59 \text { (IC } 95 \% \\
0,41-0,85 \text { ) de sintomas depressivos; } \\
\text { Mantiveram-se ativos: } \mathrm{RP}=0,75 \text { (IC } 95 \% \\
0,64-0,81 \text { ) de sintomas de ansiedade; } \\
\text { Tornaram-se ativos: } \mathrm{RP}=0,62 \text { (IC } 95 \% \\
0,47-0,81 \text { ). }\end{array}$ \\
\hline $\begin{array}{l}\text { Meira } \\
\text { Júnior et } \\
\text { al., } 2020\end{array}$ & $\begin{array}{c}\text { Todo } \\
\text { Brasil, } \\
\text { principalmente } \\
\text { a } \\
\text { região } \\
\text { Sudeste }\end{array}$ & $\begin{array}{c}571 \\
\text { adultos }\end{array}$ & $\begin{array}{l}\text { Idade média } \\
\text { de } 39 \pm 14 \\
\text { anos. }\end{array}$ & $65 \%$ & $\begin{array}{c}\text { Antes do } \\
\text { distanciamento } \\
\text { AF } \geq 3 \times \text { sem: } 75,5 \% \\
\text { AF } \geq 40 \mathrm{~min} / \text { dia: } \\
86,9 \% \\
\text { AF } \geq 3 \times / \text { sem e } \\
\geq 40 \mathrm{~min} / \text { dia: } 65,3 \% \\
\text { Durante } \\
\text { distanciamento } \\
\text { AF } \geq 3 \times \text { sem: } 67,9 \% \\
A F \geq 40 \mathrm{~min} / \text { dia: } 65,6 \% \\
\text { AF } \geq 3 \times \text { sem e } \\
\geq 40 \mathrm{~min} / \text { dia: } 47,2 \%\end{array}$ & $\begin{array}{c}\text { Autorrelato } \\
\text { pelo VIGITEL, } \\
\text { c om } 5 \text { questões. }\end{array}$ & - & STAI-S-6 & $\begin{array}{c}A F \geq 40 \mathrm{~min} / \mathrm{dia}: \beta=-1,04 \text { (IC } 95 \%-1,81 \\
\mathrm{a}-0.27) ; \\
\mathrm{AF} \geq 3 \mathrm{x} / \mathrm{sem}: \beta=-1,57 \text { (IC } 95 \%-2,32 \text { a - } \\
0,81) ; \\
\mathrm{AF} \geq 3 \mathrm{x} / \mathrm{sem} \text { e } \geq 40 \mathrm{~min} / \text { dia: } \beta=-1,78 \text { (IC } \\
95 \%-2,49 \text { a }-1,07) .\end{array}$ \\
\hline
\end{tabular}




\begin{tabular}{|c|c|c|c|c|c|c|c|c|c|}
\hline \multirow[b]{2}{*}{$\begin{array}{l}\text { AUTOR/ } \\
\text { ANO }\end{array}$} & \multirow[b]{2}{*}{$\begin{array}{l}\text { REGIÃO } \\
\text { DO PAÍS }\end{array}$} & \multicolumn{3}{|c|}{ AMOSTRA } & \multirow[b]{2}{*}{$\begin{array}{l}\text { Sexo (\% S } \\
\text { feminino) }\end{array}$} & \multirow[b]{2}{*}{$\begin{array}{l}\text { INSTRUMENTO } \\
\text { ATIVIDADE } \\
\text { FÍSICA }\end{array}$} & \multirow{2}{*}{$\begin{array}{l}\text { PREVALÊNCIA DE } \\
\text { SINTOMAS DE } \\
\text { DEPRESSÃO E } \\
\text { ANSIEDADE (D\&A) }\end{array}$} & \multirow[b]{2}{*}{$\begin{array}{l}\text { INSTRUMENTO } \\
\text { SINTOMAS } \\
\text { D\&A }\end{array}$} & \multirow[b]{2}{*}{ RESULTADOS } \\
\hline & & $\mathbf{N}$ & Idade & $\begin{array}{l}\text { Sexo (\% S } \\
\text { feminino) }\end{array}$ & & & & & \\
\hline $\begin{array}{l}\text { Schuch et } \\
\text { al. } 2020\end{array}$ & $\begin{array}{l}\text { Todo } \\
\text { Brasil }\end{array}$ & $\begin{array}{c}937 \\
\text { adultos }\end{array}$ & $\begin{array}{c}52.6 \% \text { dos } \\
\text { participantes } \\
\text { com idade de } \\
18-35 \text { anos }\end{array}$ & $72,3 \%$ & - & $\begin{array}{l}2 \text { perguntas } \\
\text { autorrelatadas }\end{array}$ & - & BDI e BAI & $\begin{array}{c}\geq 30 \mathrm{~min} \text { MVPA/dia: OR=0,71 (IC } \\
95 \%=0,53-0,96 \text { ) para depressão; } \\
\text { OR=0,72 (IC 95\%=0,54-0,96) para } \\
\text { ansiedade, e OR=0,71 (IC 95\%=0,52- } \\
\text { 0,96) para co-ocorrência de sintomas } \\
\text { de depressão e ansiedade; } \\
\geq 15 \mathrm{~min} \text { VPA/dia: depressão OR=0,60 } \\
\text { (IC } 95 \%=0,43-0,82 \text { ) para depressão; } \\
\text { OR=0,70 (IC 95\%=0,51-0,96) para } \\
\text { ansiedade, e OR=0,59 (IC 95\%=0,41- } \\
\text { 0,83) para co-ocorrência de sintomas } \\
\text { de depressão e ansiedade. }\end{array}$ \\
\hline $\begin{array}{l}\text { Silva et. } \\
\text { al, } 2020\end{array}$ & $\begin{array}{l}\text { Todo } \\
\text { Brasil }\end{array}$ & $\begin{array}{c}1.154 \\
\text { adultos }\end{array}$ & $\begin{array}{l}\text { Idade média } \\
31,15 \pm 9,68 \\
\text { anos }\end{array}$ & $69,8 \%$ & $\begin{array}{c}\text { Antes do } \\
\text { distanciamento } \\
\text { Inativos: } 30,3 \% \\
\text { Ativos }{ }^{3}: 69,7 \% \\
\text { Durante } \\
\text { distanciamento } \\
\text { Inativos: } 50,7 \% \\
\text { Ativos }{ }^{3}: 49,3 \%\end{array}$ & $\begin{array}{l}\text { Questionário } \\
\text { sobre prática de } \\
\text { exercícios } \\
\text { físicos antes e } \\
\text { durante } \\
\text { Surto de COVID- } \\
\text { 19, sem } \\
\text { especificar } \\
\text { número de } \\
\text { questões. }\end{array}$ & $\begin{array}{c}\text { Depressão: } \\
\text { Leve: } 14,1 \% \\
\text { Moderada: } 21,6 \% \\
\text { Grave: } 8,2 \% \\
\text { Muito Grave: } 10,6 \% \\
\text { Ansiedade: } \\
\text { Leve: } 6,7 \% \\
\text { Moderada: } 16,9 \% \\
\text { Grave: } 6,4 \% \\
\text { Muito Grave: } 11,1 \%\end{array}$ & DASS-21 & $\begin{array}{c}\text { Fisicamente inativos e chance de } \\
\text { depressão: OR=2,525 (IC 95\%=1,991- } \\
\text { 3,205); } \\
\text { Fisicamente inativos e chance de } \\
\text { sintomas aumentados de ansiedade: } \\
\text { OR=2,183 (IC } 95 \%=1,717-2,775) .\end{array}$ \\
\hline $\begin{array}{l}\text { Solon } \\
\text { Júnior et } \\
\text { al. } 2020\end{array}$ & $\begin{array}{l}\text { Regiões: } \\
\text { nordeste, } \\
\text { centrooeste } \\
\quad \mathrm{e} \\
\text { sudeste }\end{array}$ & $\begin{array}{c}89 \\
\text { adultos }\end{array}$ & $\begin{array}{c}\text { Média de } \\
\text { idade } 22,93 \pm \\
2,58 \text { anos }\end{array}$ & $57,3 \%$ & $\begin{array}{l}\text { Inativos }^{(4)}: 61,8 \% \\
\text { (grupos PIBC e } \\
\text { PIDC) } \\
\text { Ativos }^{(5)}: 38,2 \% \\
\text { (grupo HBE) }\end{array}$ & $\begin{array}{l}\text { Autorrelato, } \\
\text { somente } \\
\text { relataram se } \\
\text { praticavam ou } \\
\text { não AF }\end{array}$ & - & DASS-21 & $\begin{array}{l}\text { Os grupos PIBC e PIDC apresentaram } \\
\text { pontuações médias mais altas para } \\
\text { depressão }(p=0,02, E S d=0,65) \text { e } \\
\text { ansiedade }(p=0,004, E S d=0,96) \text { em } \\
\text { relação ao grupo HBE. }\end{array}$ \\
\hline
\end{tabular}

AF: Atividade Física; BAI: Beck Anxiety Inventory; BDI: Beck Depression Inventory; $\beta$ : Coeficiente beta; DASS-21: Depression, Anxiety, and Stress Scale - Short Form; ES: effect size; ESd: Cohen's "d" effect sizes; GAD-7: General Anxiety Disorder-7; IC: Intervalo de confiança; HADS: Hospital Anxiety and Depression Scale; HBE group: Grupo de exercícios em casa; IPAQ: International Physical Activity

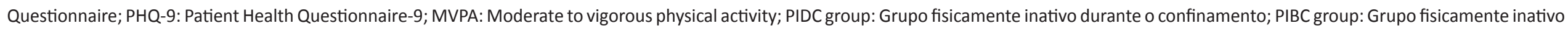
antes do confinamento; STAI-S-6: State and Trait Anxiety Inventory Brazilian short-version; VIGITEL ; Sistema de Vigilância de Fatores de Risco e Proteção para Doenças Crônicas por Inquérito Telefônico;

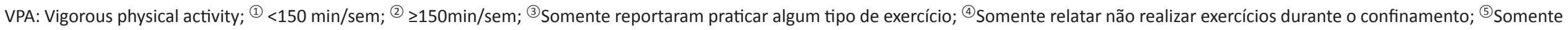
relatar exercitarem-se em casa durante. 
Um estudo transversal, que avaliou 1.154 sujeitos, identificou que pessoas que relataram que não se exercitaram durante o distanciamento social apresentaram um risco duas vezes maior de depressão $(O R=2,525$; IC $95 \%=1,991-3,205)$ e um risco duas vezes maior de sintomas de ansiedade aumentados (OR=2,183; IC 95\%= 1,717-2,775) em comparação com indivíduos que continuaram se exercitando durante o distanciamento ${ }^{18}$. Ainda, mulheres que relataram não se exercitar apresentaram um risco duas vezes maior de depressão ( $O R=2,363$; IC 95\%=1,771-3,154) e ansiedade (OR=2,341; IC 95\%=1,760-3,112); homens que relataram não se exercitar, apresentaram um risco quatro vezes maior de depressão (OR=4,045; IC 95\%=2,657-6,155) e duas vezes maior de ansiedade $(\mathrm{OR}=2,929 \text {; IC } 95 \%=1,911-4,449)^{18}$.

O estudo de Schuch et al. ${ }^{17}$, que avaliou 937 adultos, identificou que os participantes que relataram praticar 30 minutos ou mais de atividade física moderada a vigorosa por dia apresentaram um risco $29 \%$ de apresentarem sintomas depressivos ( $O R=0,71$; IC 95\%=0,53-0,96), 28\% de ansiedade $(O R=0,72$; IC 95\%=0,540,96), e $29 \%$ na co-ocorrência de sintomas de depressão e ansiedade (OR=0,71; IC 95\%=0,52-0,96). Pessoas que relataram realizar 15 minutos ou mais de atividade vigorosa por dia apresentaram $40 \%$ menos chances de depressão (OR=0,60; IC 95\%=0,43-0,82), 30\% menos chances de ansiedade (OR=0,70; IC 95\%=0,51-0,96), e $41 \%$ menos chances da co-ocorrência de sintomas de depressão e ansiedade $\left(O R=0,59\right.$, IC 95\%=0,41-0,83) ${ }^{17}$.

Homens e mulheres têm maior probabilidade de apresentar sintomas depressivos elevados quando possuem alguma condição crônica e são fisicamente inativos em comparação àqueles que não apresentam condições crônicas e são fisicamente ativos ${ }^{15}$ (Andrade-Lima et al., 2020). Além disso, homens inativos e sem condições crônicas apresentam mais chances de apresentarem sintomas depressivos elevados $(O R=1,64$, IC $95 \%=1,15-2,34)$ quando comparados a homens ativos e sem doenças crônicas ${ }^{15}$. Mulheres sem nenhuma condição crônica e fisicamente inativas apresentam uma probabilidade de 30\% a 40\% maior de apresentarem sintomas depressivos $(O R=1,16$, IC 95\%=0,87-1,54) quando comparadas a mulheres sem condições crônicas e fisicamente ativas ${ }^{15}$.

O estudo longitudinal de Feter et al. ${ }^{12}$, que acompanhou 2.314 indivíduos, encontrou uma menor prevalência (razão de prevalência [RP]=0,72; IC 95\% 0,57-0,91) de sintomas depressivos em indivíduos que relataram que se mantiveram ativos (150 minutos ou mais por semana) ou que se tornaram ativos durante a pandemia ( $\mathrm{RP}=0,59$; IC $95 \%$ 0,41-0,85) quando comparados àqueles que se mantiveram constantemente inativos. A prática de atividade física foi associada a menos sintomas de ansiedade, sendo que indivíduos que se mantiveram fisicamente ativos antes e durante o distanciamento social apresentaram prevalência $25 \%$ menor de ansiedade ( $R P=0,75$, IC 95\% 0,64-0,81) e aqueles que se tornaram fisicamente ativos apresentaram prevalência 38\% menor ( $\mathrm{RP}=0,62$, IC 95\% 0,47-0,81) de sintomas de ansiedade em comparação com indivíduos que se mantiveram inativos ${ }^{12}$.

O estudo de Solon Júnior et al. ${ }^{19}$ identificou que pessoas que eram fisicamente inativas durante o confinamento apresentaram pontuações médias mais altas na Depression, Anxiety, and Stress Scale - Short Form (DASS-21) para depressão ( $p=0,02$, Cohen's " $d$ " effect sizes $(E S d)=0,65)$ e ansiedade $(p=0,004, E S d=0,96)$ quando comparadas a pessoas que se exercitaram em casa durante o confinamento. 
No estudo transversal, realizado por Meira Júnior et al. ${ }^{16}$, com relação a prática de atividade física de lazer (considerada como a atividade física realizada por um indivíduo não como uma atividade essencial da vida diária e que é realizada a critério do indivíduo $\left.{ }^{20}\right)$, para o desfecho ansiedade, não houve diferença na pontuação média do STAI-S-6 entre os que praticavam uma vez na semana e os que não praticavam (Praticavam $(P)=15,11$ \pm 4 ; Não praticavam $(N P)=15,93 \pm 4,54 ; p=0,198$ ). Entretanto, aqueles que praticaram 40 minutos ou mais por sessão em comparação com aqueles que praticaram menos de 40 minutos por sessão $(P=14,77 \pm 4,03 ; N P=$ $15,82 \pm 3,9 ; p=0,005)$, três ou mais dias por semana em comparação com aqueles que praticavam menos de 3 dias por semana ( $P=14,57 \pm 3,94 ; N P=16,33 \pm 3,88 ; p<0,0001)$, e aqueles que realizaram pelo menos 40 minutos por sessão em três ou mais dias da semana em comparação com aqueles que praticaram 2 vezes ou menos e também com os que não praticavam nada ( $P=15,87 \pm 4,09, N P=14,3 \pm 3,9 ; p<0,0001)$ apresentaram níveis significativamente mais baixos de ansiedade ${ }^{16}$. Não foram encontradas diferenças significativas entre aqueles que praticavam atividade física moderada com aqueles que praticavam atividade física moderada e vigorosa $(15,48 \pm 4,19 ; 14,85 \pm 3,85 ; p=0.119 \text {, respectivamente })^{16}$. Além disso, com base em modelos de regressão, ansiedade-estado, durante a pandemia, pode ser prevista pela duração da atividade física (40 minutos ou mais por dia: Coeficiente Beta $(\beta)=-1,04$; IC 95\% -1,81 a -0,27), frequência (três ou mais dias por semana: $\beta=-1,57$; IC 95\% -2,32 a -0,81) e a combinação de duração e frequência da atividade física (40 minutos ou mais por dia, três vezes por semana: $\beta=-1,78$; IC $95 \%-2,49$ a $-1,07)^{16}$. Ainda, quanto mais atividade física é realizada, menos sintomas de ansiedade estão presentes, independente de sexo, idade, nível de escolaridade, ansiedade-traço e atividade física antes da pandemia ${ }^{16}$.

\section{Discussão}

Dos estudos analisados, quatro demonstraram que há uma associação entre atividade física e sintomas de depressão e ansiedade, um demonstrou que há associação entre atividade física e depressão e um que há associação entre atividade física e sintomas de ansiedade. Os achados do presente estudo corroboram estudos anteriores a pandemia na qual a atividade física é um fator protetor contra a incidência de depressão $0^{13} \mathrm{e}$ ansiedade ${ }^{14}$, bem como, uma estratégia terapêutica para a redução dos sintomas depressivos ${ }^{21}$ e de ansiedade ${ }^{22}$.

A presente revisão demonstra com dados de estudos feitos no Brasil que, mesmo em um contexto pandêmico, contexto disruptivo quanto ao comportamento e associado a uma elevação na prevalência e incidência de transtornos mentais, a prática de atividade física está associada a uma menor presença de sintomas de depressão e ansiedade. Os achados são relevantes em termos de saúde pública pois sugerem que a promoção de atividade física pode ser uma estratégia segura e de baixo custo para mitigar o impacto da pandemia na saúde mental da população brasileira ${ }^{23}$. É preciso considerar também que problemas de saúde mental podem se apresentar principalmente entre pessoas vulneráveis e com fatores de risco, e que muitos casos podem ficar sem diagnóstico ou sem tratamento em decorrência do fechamento e da interrupção das atividades dos serviços de saúde mental24,25. Interessantemente, estudos de randomização mendeliana pré-pandemia sugerem que a atividade física parece ser protetora mesmo em pessoas com vulnerabilidade genética para a depressão ${ }^{26}$. 
A atividade física pode estar associada a menor presença de sintomas de depressão e ansiedade pois a atividade física pode promover o aumento da expressão do fator neurotrófico derivado do cérebro - o BDNF (Brain-derived neurotrophic factor); pela maior disponibilidade de serotonina e norepinefrina, pela regulação da atividade do eixo Hipotálamo-Pituitária-Adrenal (HPA) e ainda pela redução da inflamação sistêmica ${ }^{27,28,29}$. Ainda, o exercício físico, que é a atividade física estruturada e sistematizada promove alterações estruturais em regiões subcorticais associadas com a depressão, como o hipocampo ${ }^{30}$. Existem ainda benefícios psicossociais proporcionados pela atividade física que podem contribuir para a melhora dos sintomas de depressão e ansiedade, como a melhora da autoestima, que pode colaborar na melhora do humor; melhora do apoio social, considerando que o exercício pode ser uma forma de socialização, o que pode contribuir para melhora de sintomas depressivos; e ainda a melhora da autoeficácia, que está relacionada à autoconfiança específica da situação, ou seja, quando o indivíduo acredita que pode realizar uma tarefa específica ${ }^{31}$. Além de contribuir na melhora da depressão e ansiedade, a atividade física pode colaborar na prevenção de recidivas dos sintomas, é também benéfica para outras comorbidades que o indivíduo possa apresentar, sendo ainda uma estratégia de baixo custo e acessível ${ }^{32}$.

\section{Limitações}

O presente estudo tem algumas limitações. Não foi possível realizar uma meta-análise devido à heterogeneidade dos tratamentos estatísticos realizados dos estudos incluídos. Além disso, a maioria dos estudos incluídos são transversais, sendo assim, há impossibilidade de realizar interferências causais. Ainda, somente um estudo utilizou uma amostra representativa da população brasileira, e, dessa forma, classes econômicas mais baixas, pessoas com menor nível de escolaridade e homens podem estar sub-representados nos demais estudos incluídos.

\section{Conclusão}

Brasileiros que praticaram atividade física durante o período de distanciamento social tiveram menos chances de apresentar sintomas de ansiedade e depressão em relação a pessoas que não realizaram atividade física durante o isolamento. Realizar exercícios físicos durante o período de distanciamento físico pode ser uma estratégia benéfica para promoção da saúde tanto física quanto mental. Cabe destacar que, na realização dos exercícios, devem-se respeitar as medidas preconizadas, realizando exercícios individualmente ou em duplas, mantendo distanciamento social, evitando aglomerações e mantendo hábitos de higiene individual (respiratória e das mãos) e do ambiente (higienização de equipamentos de uso comum, ventilação).

\section{Referências}

1. WORLD HEALTH ORGANIZATION. Coronavírus disease (COVID-19) Pandemic. 2020. Disponível em: https:// www. who.int/emergencies/diseases/novel-coronavirus-2019. Acesso em: 26 jan. 2021.

2. Ministerio da Saúde. Boletim epidemiológico 07-06/04/2020. In: Saude Md, ed. In: https://portalarquivos. 
saude.gov.br/images/pdf/2020/April/06/2020-04-06-BE7-Boletim- Especial-do-COE-Atualizacao-daAvaliacao-de-Risco.pdf. Acesso em 26 jan. 2021

3. Carvalho Malta D, Saar Gomes C, Landmann Szwarcwald C, Berti de Azevedo Barros M, Gomes da Silva A, Junio Sady Prates E et al. Distanciamento social, sentimento de tristeza e estilos de vida da população brasileira durante a pandemia de COVID-19. SciELO Preprints "No prelo" 2020. doi: 10.1590/ SciELOPreprints.1371.

4. Stockwell S, Trott M, Tully M, Shin J, Barnett $Y$, Butler $L$ et al. Changes in physical activity and sedentary behaviours from before to during the COVID-19 pandemic lockdown: a systematic review. BMJ Open Sport \& Exercise Medicine. 2021;7(1):e000960. doi: 10.1136/bmjsem-2020- 000960.

5. Costa C, Costa T, Barbosa Filho V, Bandeira P, Siqueira R. Influência do distanciamento social no nível de atividade física durante a pandemia do COVID-19. Revista Brasileira de Atividade Física \& Saúde. 2020;25:16.

6. Malta D, Szwarcwald C, Barros M, Gomes C, Machado Í, Souza Júnior P et al. A pandemia da COVID-19 e as mudanças no estilo de vida dos brasileiros adultos: um estudo transversal, 2020. Epidemiologia e Serviços de Saúde. 2020;29(4).

7. Marques M, Gheller R, Henrique N, Menezes E, Streit I, Franchini E et al. Physical activity during the COVID-19 pandemic: a survey with adults in Northern Brazil. Revista Brasileira de Atividade Física \& Saúde. 2020;25:1-8.

8. Ornell F, Schuch J, Sordi A, Kessler F. "Pandemic fear" and COVID-19: mental health burden and strategies. Brazilian Journal of Psychiatry. 2020;42(3):232-235. https://doi.org/10.1590/1516-4446-2020-0008

9. Arora T, Grey I, Östlundh L, Lam K, Omar O, Arnone D. The prevalence of psychological consequences of COVID-19: A systematic review and meta-analysis of observational studies. Journal of Health Psychology. 2020;:135910532096663. doi: 10.1177/1359105320966639.

10. Xiong J, Lipsitz O, Nasri F, Lui L, Gill H, Phan L et al. Impact of COVID-19 pandemic on mental health in the general population: A systematic review. Journal of Affective Disorders. 2020;277:55-64. doi: 10.1016/j. jad.2020.08.001.

11. Barros M, Lima M, Malta D, Szwarcwald C, Azevedo R, Romero D et al. Relato de tristeza/depressão, nervosismo/ansiedade e problemas de sono na população adulta brasileira durante a pandemia de COVID-19. Epidemiologia e Serviços de Saúde. 2020;29(4).

12. Feter N, Caputo E, Doring I, Leite J, Cassuriaga J, Reichert F et al. Sharp increase in depression and anxiety among Brazilian adults during the COVID-19 pandemic: findings from the PAMPA cohort. Public Health. 2021;190:101-107.

13. Schuch F, Vancampfort D, Firth J, Rosenbaum S, Ward P, Silva E et al. Physical Activity and Incident Depression: A Meta-Analysis of Prospective Cohort Studies. American Journal of Psychiatry. 2018;175(7):631-648.

14. Schuch F, Stubbs B, Meyer J, Heissel A, Zech P, Vancampfort D et al. Physical activity protects from incident anxiety: A meta-analysis of prospective cohort studies. Depression and Anxiety. 2019;36(9):846-858. doi: 10.1002/da.22915.

15. Andrade-Lima A, Werneck A, Szwarcwald C, Schuch F, Stubbs B, Bastos A et al. The role of physical activity in the association between multimorbidity and depressive symptoms: Data from 60,202 adults from the Brazilian National Health Survey. Journal of Psychosomatic Research. 2020;134:110122.

16. Meira C, Meneguelli K, Leopoldo M, Florindo A. Anxiety and Leisure-Domain Physical Activity Frequency, Duration, and Intensity During Covid-19 Pandemic. Frontiers in Psychology. 2020;11. doi: 10.3389/ fpsyg.2020.603770. 
17. Schuch F, Bulzing R, Meyer J, Vancampfort D, Firth J, Stubbs B et al. Associations of moderate to vigorous physical activity and sedentary behavior with depressive and anxiety symptoms in self-isolating people during the COVID-19 pandemic: A cross-sectional survey in Brazil. Psychiatry Research. 2020;292:113339. doi: 10.1016/j.psychres.2020.113339.

18. Silva L, Seguro C, de Oliveira C, Santos P, de Oliveira J, de Souza Filho L et al. Physical Inactivity Is Associated With Increased Levels of Anxiety, Depression, and Stress in Brazilians During the COVID-19 Pandemic: A Cross-Sectional Study. Frontiers in Psychiatry. 2020;11. doi: 10.3389/fpsyt.2020.565291.

19. Solon Júnior L, Fortes L, Barbosa B, Feitosa Júnior J, Ribeiro C, Silva Neto L. Home-based exercise during confinement in COVID-19 pandemic and mental health in adults: a crosssectional comparative study. Revista Brasileira de Atividade Física \& Saúde. 2020;25:1-7.

20. Bull F, Al-Ansari S, Biddle S, Borodulin K, Buman M, Cardon G et al. World Health Organization 2020 guidelines on physical activity and sedentary behaviour. British Journal of Sports Medicine. 2020;54(24):1451-1462. doi: 10.1136/bjsports-2020-102955.

21. Schuch F, Vancampfort D, Richards J, Rosenbaum S, Ward P, Stubbs B. Exercise as a treatment for depression: A meta-analysis adjusting for publication bias. Journal of Psychiatric Research. 2016;77:42-51. doi: 10.1016/j. jpsychires.2016.02.023.

22. Stubbs B, Vancampfort $D$, Rosenbaum S, Firth J, Cosco T, Veronese $N$ et al. An examination of the anxiolytic effects of exercise for people with anxiety and stress-related disorders: A metaanalysis. Psychiatry Research. 2017;249:102-108.doi:10.1016/j.psychres.2016.12.020.

23. Souza Filho B, Tritany É. COVID-19: importância das novas tecnologias para a prática de atividades físicas como estratégia de saúde pública. Cadernos de Saúde Pública. 2020;36(5). doi: 10.1590/0102$311 \times 00054420$.

24. Sergeant A, van Reekum EA, Sanger N, Dufort A, Rosic T, Sanger S et al. Impact of COVID-19 and other pandemics and epidemics on people with pre-existing mental disorders: a systematic review protocol and suggestions for clinical care. BMJ Open. 2020;10:e040229. doi:10.1136/bmjopen-2020-040229.

25. Vadivel R, Shoib S, El Halabi S, El Hayek S, Essam L, Gashi Bytyçi D et al. Mental health in the postCOVID-19 era: challenges and the way forward. General Psychiatry. 2021;34(1):e100424. doi:10.1136/ gpsych-2020-100424.

26. Choi K, Zheutlin A, Karlson R, Wang M, Dunn E, Stein M et al. Physical activity offsets genetic risk for incident depression assessed via electronic health records in a biobank cohort study. Depression and Anxiety. 2019;37(2):106-114. doi: 10.1002/da.22967.

27. Schuch F, Vancampfort D, Rosenbaum S, Richards J, Ward P, Veronese $\mathrm{N}$ et al. Exercise for depression in older adults: a meta-analysis of randomized controlled trials adjusting for publication bias. Revista Brasileira de Psiquiatria. 2016;38(3):247-254. doi: 10.1590/1516-4446- 2016-1915.

28. Gujral S, Aizenstein H, Reynolds C, Butters M, Erickson K. Exercise effects on depression: Possible neural mechanisms. General Hospital Psychiatry. 2017;49:2-10. doi: 10.1016/j.genhosppsych.2017.04.012.

29. Hu S, Tucker L, Wu C, Yang L. Beneficial Effects of Exercise on Depression and Anxiety During the Covid-19 Pandemic: A Narrative Review. Frontiers in Psychiatry. 2020;11. doi:10.3389/fpsyt.2020.587557.

30. Firth J, Stubbs B, Vancampfort D, Schuch F, Lagopoulos J, Rosenbaum S et al. Effect of aerobic exercise on hippocampal volume in humans: A systematic review and meta-analysis. Neurolmage. 2018;166:230-238.. doi: 10.1016/j.neuroimage.2017.11.007.

31. Kandola A, Ashdown-Franks G, Hendrikse J, Sabiston C, Stubbs B. Physical activity and depression: Towards understanding the antidepressant mechanisms of physical activity. Neuroscience \& Biobehavioral Reviews. 2019;107:525-539. doi: 10.1016/j.neubiorev.2019.09.040. 
32. Schmitter M, Spijker J, Smit F, Tendolkar I, Derksen A, Oostelbos P et al. Exercise enhances: study protocol of a randomized controlled trial on aerobic exercise as depression treatment augmentation. BMC Psychiatry. 2020;20(1). doi: 10.1186/s12888-020-02989-z.

Contribuições: Aline Josiane Waclawovsky - Análise estatística, Coleta de Dados, Conceitualização, Investigação, Metodologia, Redação - Preparação do original, Redação - Revisão e Edição;

Eduarda Bitencourt dos Santos - Coleta de Dados, Conceitualização, Redação - Preparação do original, Redação

- Revisão e Edição;

Felipe Barreto Schuch - Gerenciamento do Projeto, Metodologia, Redação - Preparação do original, Redação - Revisão e Edição, Supervisão.

\section{Correspondência}

Aline Josiane Waclawovsky

alinejw@yahoo.com.br

Submetido em: 01/03/2021

Aceito em: 14/04/2021 